\title{
Energy Cost of Activities in Preschool-Aged Children
}

\author{
Maurice R. Puyau, Anne L. Adolph, Yan Liu, Theresa A. Wilson, Issa F. Zakeri, and Nancy F. Butte
}

\begin{abstract}
Background: The absolute energy cost of activities in children increases with age due to greater muscle mass and physical capability associated with growth and developmental maturation; however, there is a paucity of data in preschool-aged children. Study aims were 1) to describe absolute and relative energy cost of common activities of preschool-aged children in terms of $\mathrm{VO}_{2}$, energy expenditure (kilocalories per minute) and child-specific metabolic equivalents (METs) measured by room calorimetry for use in the Youth Compendium of Physical Activity, and 2) to predict METs from age, sex and heart rate (HR). Methods: Energy expenditure (EE), oxygen consumption $\left(\mathrm{VO}_{2}\right), \mathrm{HR}$, and child-METs of 13 structured activities were measured by room respiration calorimetry in 119 healthy children, ages 3 to 5 years. Results: EE, $\mathrm{VO}_{2}$, $\mathrm{HR}$, and child-METs are presented for 13 structured activities ranging from sleeping, sedentary, low-, moderate- to high-active. A significant curvilinear relationship was observed between child-METs and HR $\left(r^{2}=.85 ; P=.001\right)$. Conclusion: Age-specific child METs for 13 structured activities in preschool-aged children will be useful to extend the Youth Compendium of Physical Activity for research purposes and practical applications. HR may serve as an objective measure of MET intensity in preschool-aged children.
\end{abstract}

Keywords: energy expenditure, oxygen consumption, child-METs, heart rate

The absolute energy cost of activities in children increases with age due to greater muscle mass and physical capability associated with growth and developmental maturation. ${ }^{1}$ Physical activity level (PAL), defined as the ratio of total energy expenditure to basal metabolic rate (BMR), also increases from early infancy to early childhood to adolescence, primarily due to the decreasing BMR. ${ }^{2}$ The inappropriate use of adult values for the energy cost of activities, expressed as metabolic equivalents (METs) or PAL, in children is well recognized. ${ }^{3,4}$ This difference in the energy cost of activities is greatest in very young children and was first recognized by Torun et al in children 2 to 4 years of age. ${ }^{3}$

Measurements of the energy expenditure (EE) of physical activities in preschool-aged children, however, are sparse. Although $\mathrm{EE}$ in preschoolers has been measured using portable calorimeters in an effort to calibrate accelerometers, ${ }^{5,6}$ the energy cost of only a few activities were reported. EE has also been assessed using a room respiration calorimeter, but the energy cost were not reported by age and/or activity. ${ }^{7,8}$ The paucity of EE data in this age group is due to many obstacles including child cooperation, limited comprehension, short attention span, physical ability, and available measuring devices. In addition, indirect measurements of EE are sparse in preschoolers. Based on the linear relationship between EE and HR over a range of activities in adults, ${ }^{9} \mathrm{HR}$ may serve as a good proxy measure of EE and METs in preschoolers.

The aims of this study were 1) to describe the energy cost of common activities of preschool-aged children in terms of childspecific METs measured in a child-friendly room respiration calorimeter for use in the Youth Compendium of Physical Activity, and 2) to predict METs from age, sex and heart rate (HR).

Puyau (mpuyau@bcm.edu), Adolph, Liu, Wilson, and Butte are with the Dept of Pediatrics, Baylor College of Medicine. Zakeri is with the Dept of Epidemiology and Biostatistics, Drexel University.

\section{Methods}

\section{Participants}

Participants were 119 healthy 3- to 5-year-olds (59 boys and 60 girls; mean age $4.5 \pm 0.9$ years; 29\%, Caucasian, $29 \%$ African American; $34 \%$ Hispanic, and $8 \%$ other). Children on prescription drugs or with chronic diseases including metabolic or endocrine disorders, asthma treated with steroids, sleep apnea, and any condition that interfered with physical activity were excluded from the study.

\section{Study Design}

The protocol entailed a 7-hour visit to the Children's Nutrition Research Center metabolic research unit. The child was instructed to follow a protocol designed to characterize EE of activities typical of this age group while in a child-friendly room respiration calorimeter.

The children were recruited using fliers at local clinics and preschool centers in Houston. The Institutional Review Board for Human Subject Research for Baylor College of Medicine and Affiliated Hospitals approved the protocol.

Anthropometry. Body weight to the nearest $0.1 \mathrm{~kg}$ was measured with a digital scale and height to the nearest $1 \mathrm{~mm}$ was measured with a stadiometer. BMI was calculated as weight/height ${ }^{2}$ $\left(\mathrm{kg} / \mathrm{m}^{2}\right)$.

Calorimetry. Oxygen consumption $\left(\mathrm{VO}_{2}\right)$ and carbon dioxide production $\left(\mathrm{VCO}_{2}\right)$ were measured continuously in a $19 \mathrm{~m}^{3}$ fast response room calorimeter, ${ }^{10}$ following a structured protocol. ${ }^{11} \mathrm{EE}$ was computed using the Weir equation. During calorimetry, HR was measured by the Actiheart device (CamNtech Ltd, Cambridge, UK) affixed on the chest using 2 electrodes (Skintact Premier, Leonhard Lang GmbH, Innsbruck, Austria). $\mathrm{VO}_{2}, \mathrm{VCO}_{2}, \mathrm{EE}$, and $\mathrm{HR}$ were averaged at 1-min intervals. Basal metabolic rate (BMR) was calculated using Schofield age- and sex-specific equations. ${ }^{12}$ Child-MET was calculated as EE/BMR. 


\section{Structured Activities}

Before measurement, the children were habituated to the calorimeter that was decorated as a playroom with age-appropriate furniture and toys. While in the calorimeter, all children were asked to voluntarily perform a series of familiar age-appropriate activities in the same order between 9:00 AM and 4:00 PM under staff direction and supervision. All measurements were performed while the children were in the fed state. The staff recorded minute-to-minute observations of the child's activities and whether the child kept to the task assigned. During the morning and afternoon sessions activities were scheduled from low to higher EE. Time spent at each activity varied due to the interest and age of the child. The calorimeter protocol included the following discrete activities: Sleep: Napping - children slept on a child's bed in the calorimeter for $65 \pm 23$ minutes after lunch; Sedentary: Watching TV sitting - children reclined against a pillow and watched a cartoon or movie on TV for $18 \pm 6$ minutes. Children were reminded via intercom to remain seated; Coloring-children sat in a chair at a desk drawing with crayons for $12 \pm 5$ minutes; Video games - children played video games while sitting on a chair for $14 \pm 15$ minutes; Puzzles - children assembled puzzles while sitting on the floor for $12 \pm 5$ minutes; Low active play: Kitchen/ toys - children played at a child's kitchen or with other toys while standing and/or kneeling for $22 \pm 10$ minutes; Moderate active play: Dance-children performed a variety of dance movements following a video for $11 \pm 3$ minutes; Active video game-children played a variety of motion games while standing on a video game mat for $10 \pm 5$ minutes; Motor skills and aerobics-children performed a variety of motor skills and aerobic activities following a video for $12 \pm 4$ minutes; Walking self-paced-children transferred balls from bucket to bucket across the calorimeter while walking for $7 \pm 4$ minutes; Ball toss (Dodgeball) — children repeatedly threw balls at targets across the room and walked quickly to retrieve the balls for $10 \pm 5$ minutes; Jogging in place - children jogged in place while competing in a video game race for $5 \pm 2$ minutes; Very active play: Running in place-children ran in place while competing in a video game race for $4 \pm 1$ minutes.

\section{Statistics}

Descriptive statistics and graphical methods (ie, histograms and scattergrams) were used to analyze the data. Adherence to the task assigned was evaluated minute to minute via the written record kept by the research staff. Only activities that were performed for 4 minutes or more of sustained activity and/or steady state EE were included in the summary statistics. Means and standard deviations for $\mathrm{EE}, \mathrm{VO}_{2}, \mathrm{HR}$, and child-METs were calculated for each verified structured activity. ANOVA was used to investigate the impact of child characteristics (age and sex) on EE of the structured activities. Tukey-Kramer's post hoc significance tests were applied for multiple comparisons between age groups. A curvilinear equation was developed for the relationship between child METs and HR. Statistical analyses were performed in SPSS (version 23.0. Armonk, NY) and SAS (version 9.4; Cary, NC).

\section{Results}

Descriptive characteristics of the 119 preschool-aged children are summarized in Table 1. On average, each child completed $9 \pm 2$ of the 13 structured activities. Missing activities were mainly due to refusal, and/or failure to understand instructions or stay on task. About half $(n=61)$ of the children took naps. $\mathrm{VO}_{2}, \mathrm{EE}, \mathrm{HR}$, and child METs of the structured activities are summarized in Tables 2 and 3.

For the 13 structured activities, there were no significant differences in MET values between boys and girls. For moderate-vigorous activities, there were significant differences in MET values between ages, with the younger children attaining lower values $(P<.05)$.

A significant curvilinear relationship was observed between child-METs and $\mathrm{HR}$ and $\mathrm{HR}^{2}(P=.001)$, although $\mathrm{HR}^{2}$ made a minor contribution to the variance in child-MET.

(1)

(2)

$$
\text { Child MET }=-3.131-0.142 \text { Sex }+0.014 \text { Age }+0.039 \text { HR }
$$

$$
\text { (adjusted } r^{2}=0.846 \text { ) }
$$

$$
\begin{gathered}
\text { Child MET }=-2.182-0.140 \text { Sex }+0.014 \text { Age }+0.025 \mathrm{HR}+ \\
0.000054 \mathrm{HR}^{2}\left(\text { adjusted } r^{2}=0.848\right)
\end{gathered}
$$

where HR is in bpm, sex is coded as 1 = boys, 2 = girls; and age is in months.

\section{Discussion}

This description of the energy cost of activities in preschool-age children contributes to the development of a compendium of youth energy expenditure. ${ }^{4}$ A compendium is useful for translating physical activity questionnaires into EE and estimating the PAL of youth. The published Compendium ${ }^{13}$ provided MET values for over 200 activities in children 6 to 17.9 years of age. Approximately $35 \%$ of the MET values were derived in youth, and the remainder estimated from adult values. While there is consensus that child-METs should be used for the update of the Compendium, ${ }^{4}$ the age range also should be extended to include preschool-aged children for research

\begin{tabular}{|c|c|c|c|c|c|c|c|}
\hline & $\mathrm{n}$ & Age (y) & Height (cm) & Weight (kg) & BMI $\left(\mathrm{kg} \cdot \mathrm{m}^{-2}\right)$ & $\begin{array}{c}\text { BMI } \\
\text { (percentile) }\end{array}$ & $\begin{array}{l}\text { Predicted BMR } \\
\text { (kcal/min) }\end{array}$ \\
\hline All & 119 & $4.5 \pm 0.9$ & $106.6 \pm 7.5$ & $18.2 \pm 3.6$ & $15.8 \pm 1.6$ & $51.5 \pm 28.1$ & $0.61 \pm 0.06$ \\
\hline Boys & 59 & $4.5 \pm 0.9$ & $106.9 \pm 7.6$ & $18.3 \pm 3.4$ & $15.9 \pm 1.3$ & $52.8 \pm 26.8$ & $0.63 \pm 0.05$ \\
\hline Girls & 60 & $4.6 \pm 0.9$ & $106.3 \pm 7.4$ & $18.0 \pm 3.9$ & $15.8 \pm 1.9$ & $50.2 \pm 29.4$ & $0.59 \pm 0.05$ \\
\hline 3 years old & $39(22 \mathrm{M}, 17 \mathrm{~F})$ & $3.5 \pm 0.3$ & $99.4 \pm 4.4$ & $15.7 \pm 2.0$ & $15.9 \pm 1.1$ & $52.4 \pm 31.3$ & $0.58 \pm 0.04$ \\
\hline 4 years old & $41(18 \mathrm{M}, 23 \mathrm{~F})$ & $4.5 \pm 0.3$ & $107.5 \pm 5.5$ & $18.4 \pm 3.5$ & $15.8 \pm 1.9$ & $55.1 \pm 28.8$ & $0.61 \pm 0.05$ \\
\hline 5 years old & 39 (19 M, 20F) & $5.6 \pm 0.3$ & $112.9 \pm 5.3$ & $20.3 \pm 3.6$ & $15.9 \pm 1.9$ & $46.8 \pm 23.6$ & $0.65 \pm 0.06$ \\
\hline
\end{tabular}
purposes and practical applications such as in child-care settings and feeding programs.

Table 1 Characteristics of the Preschool-Aged Children (Mean \pm SD)

Abbreviations: BMI, body mass index; BMR, basal metabolic rate. 


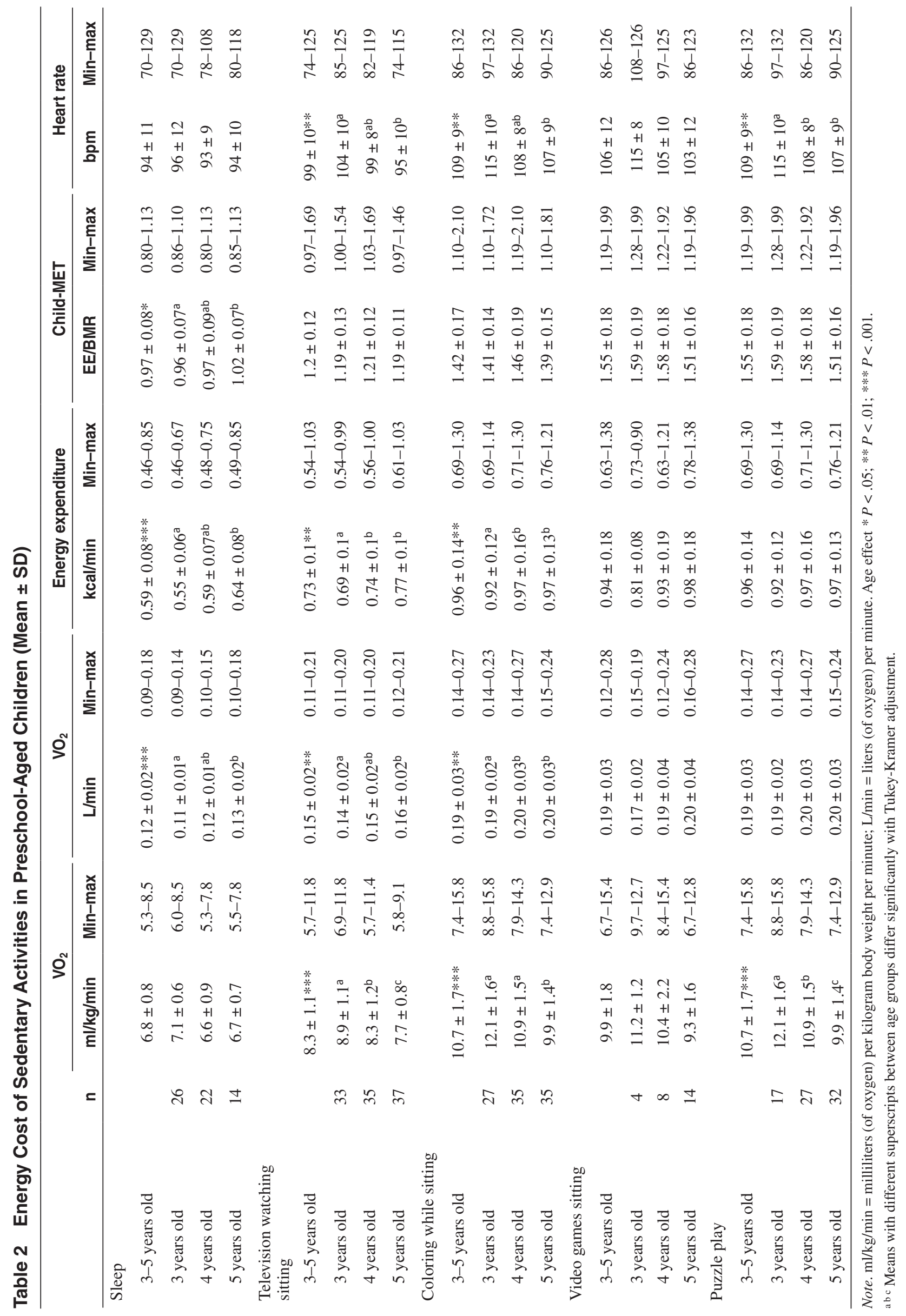




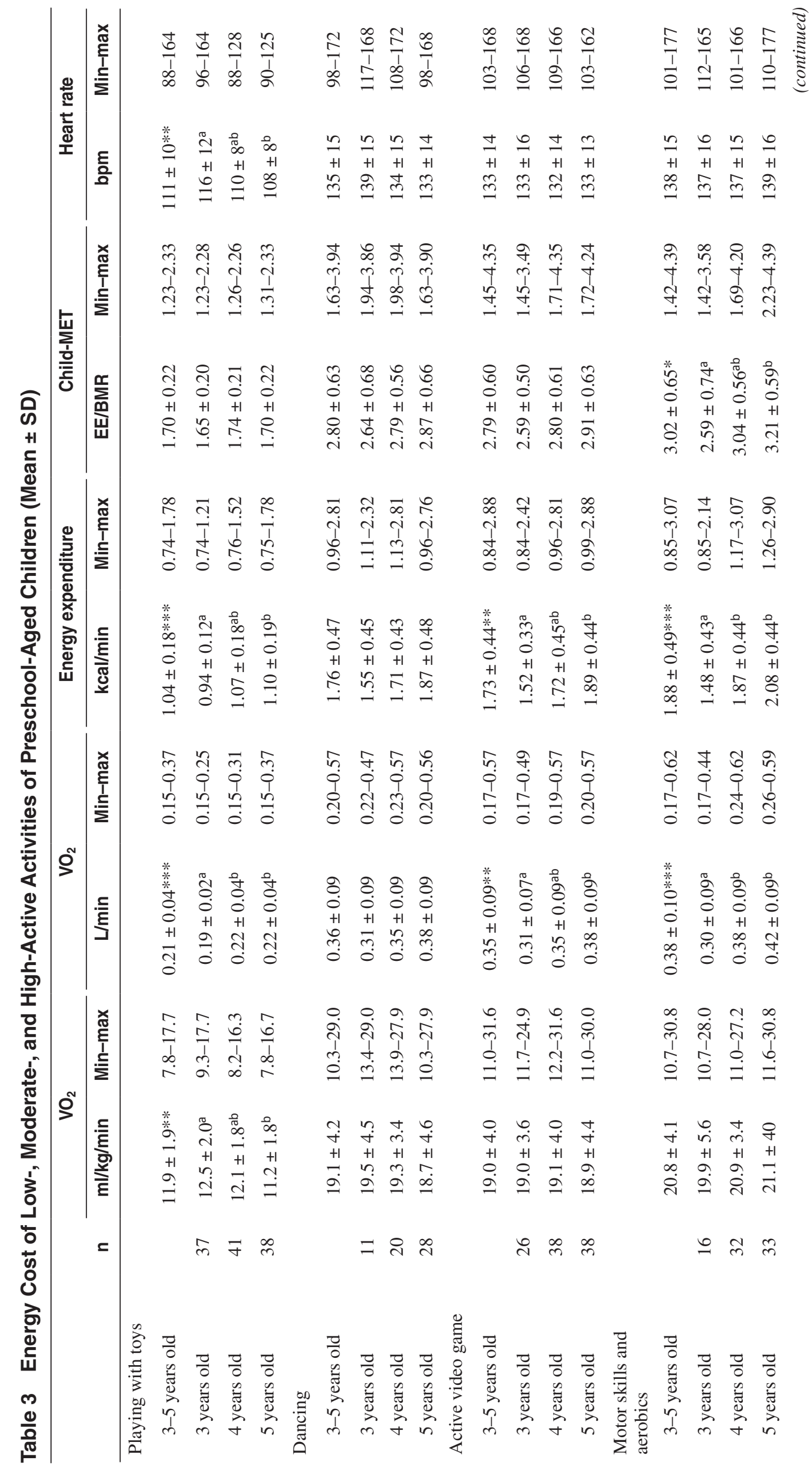




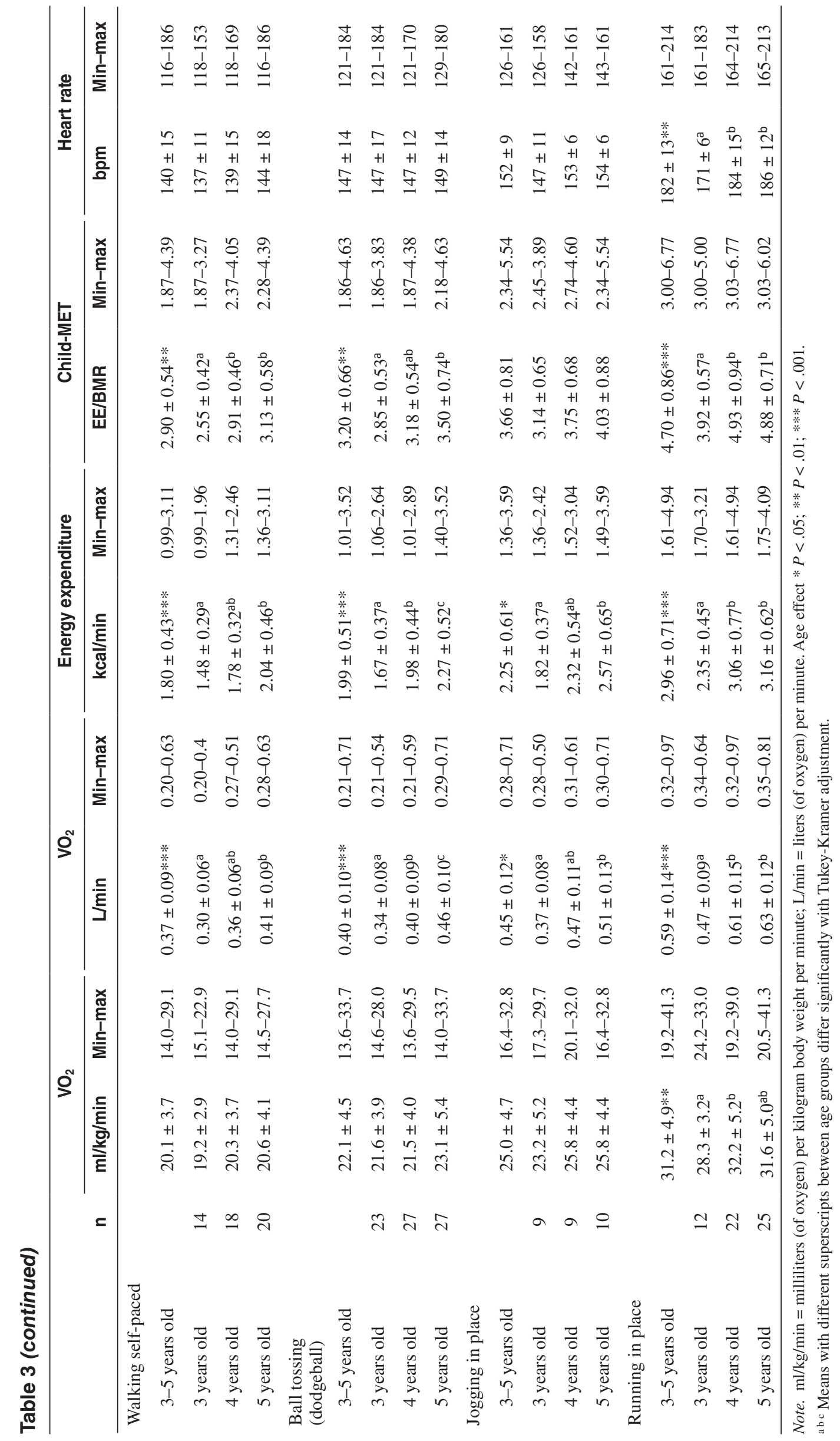


Our measurements of the energy cost of activities in preschoolaged children are comparable to other reports in preschool-aged children. ${ }^{5,14-16}$ However, the observed MET values for moderateto-vigorous physical activities are lower than older children, ${ }^{4}$ due to their higher BMRs and developmental immaturity. The mean MET values for dance, aerobics, walking and running were 2.8, 3.0, 2.9, and 4.7 in preschoolers compared with $3.5,3.8,5.0$, and 8.8 in older children ${ }^{4}$. The highest individual MET value observed in this study was 6.8 , with a corresponding $\mathrm{EE}$ of $4.9 \mathrm{kcal} / \mathrm{min}, \mathrm{VO}_{2}$ of 39.1 $\mathrm{ml} / \mathrm{kg} / \mathrm{min}$, and HR of $205 \mathrm{bpm}$ while running in place. Although these activities were not designed to measure the children's maximum effort, they were typical of preschool-aged children and did approach peak HR.

The curvilinear relationship between child METs and HR demonstrates the physiological range achievable by these young children. The Child MET prediction equation based on HR may provide a useful tool for calculating energy cost of activities in preschool aged children. Oftentimes in observational or interventional studies of physical activity, it is difficult to judge the intensity of a given activity; HR could be used as an objective measure of the intensity and corresponding MET level.

The strength of this study is accurate EE measurement of 13 relevant structured activities in a large sample of healthy preschoolaged children balanced by age, sex, and race/ethnicity. Because room calorimetry was used, the children were not burdened by mouth pieces, nose clips or gas collection apparatus for measurement of respiratory gas exchange. Limitations of the study include: performance of any given activity depended on the willingness and skill level of the child; the speed of walking, jogging, and running was not measured; and the activities in the study protocol were limited to the confines of the room calorimeter.

In conclusion, age-specific child METs for 13 structured activities in preschool-aged children will be useful to extend the Youth Compendium of Physical Activity for research purposes and practical applications. HR may serve as an objective measure of MET intensity in preschool-aged children.

\section{Acknowledgments}

The authors wish to acknowledge the contributions of the participants, Lauren DeBolt, Nitesh Mehta, MS for laboratory assistance and Janice Betancourt, RN, for nursing. This study was funded by USDA/ARS 58-6250-0-008 and NIH Grant R01 DK085163. This work is a publication of the U.S. Department of Agriculture (USDA)/Agricultural Research Service (ARS) Children's Nutrition Research Center, Department of Pediatrics, Baylor College of Medicine and Texas Children's Hospital, Houston, TX, U.S.A. The contents of this publication do not necessarily reflect the views or policies of the USDA, nor does mention of trade names, commercial products, or organizations imply endorsement by the U.S. Government.

\section{References}

1. Gabel L, Obeid J, Nguyen T, Proudfoot NA, Timmons BW. Short-term muscle power and speed in preschoolers exhibit stronger tracking than physical activity. Appl Physiol Nutr Metab. 2011;36(6):939-945. PubMed doi:10.1139/h11-118
2. Butte NF, Wong WW, Wilson TA, Adolph AL, Puyau MR, Zakeri IF. Revision of dietary reference intakes for energy in preschool-age children. Am J Clin Nutr. 2014;100(1):161-167. PubMed doi:10.3945/ ajen.113.081703

3. Torun B, Chew F, Mendoza RD. Energy cost of activities of preschool children. Nutrition R. 1983;3:401-406 Ref Type: Journal (Full). doi:10.1016/S0271-5317(83)80002-5

4. McMurray RG, Butte NF, Crouter SE, et al. Exploring metrics to express energy expenditure of physical activity in youth. PLoS One. 2015;10(6):e0130869. PubMed doi:10.1371/journal.pone.0130869

5. Pate RR, Almeida MJ, McIver KL, Pfeiffer KA, Dowda M. Validation and calibration of an accelerometer in preschool children. Obesity (Silver Spring). 2006;14(11):2000-2006. PubMed doi:10.1038/ oby. 2006.234

6. Pfeiffer KA, McIver KL, Dowda M, Almeida MJ, Pate RR. Validation and calibration of the Actical accelerometer in preschool children. Med Sci Sports Exerc. 2006;38(1):152-157. PubMed doi:10.1249/01. mss.0000183219.44127.e7

7. Janssen X, Cliff D, Okely AD, et al. Practical utility and reliability of whole-room calorimetry in young children. Br J Nutr. 2013;109:1917-1922 Ref Type: Journal (Full). PubMed doi:10.1017/ S0007114512003820

8. Adolph AL, Puyau MR, Vohra FA, Nicklas TA, Zakeri IF, Butte NF. Validation of uniaxial and triaxial accelerometers for the assessment of physical activity in preschool children. J Phys Act Health. 2012;9(7):944-953. PubMed

9. Leonard WR. Measuring human energy expenditure: what have we learned from the flex-heart rate method? Am J Hum Biol. 2003;15(4):479-489. PubMed doi:10.1002/ajhb.10187

10. Moon JK, Vohra FA, Valerio Jimenez OS, Puyau MR, Butte NF. Closed-loop control of carbon dioxide concentration and pressure improves response of room respiration calorimeters. J Nutr. 1995;125:220-228. PubMed

11. Zakeri IF, Adolph AL, Puyau MR, Vohra FA, Butte NF. Cross-sectional time series and multivariate adaptive regression splines models using accelerometry and heart rate predict energy expenditure of preschoolers. J Nutr. 2013;143(1):114-122. PubMed doi:10.3945/jn.112.168542

12. Schofield WN, Schofield C, James WPT. Basal metabolic rate-review and prediction, together with an annotated bibliography of source material. Hum Nutr Clin Nutr. 1985;39C:1-96.

13. Ridley K, Ainsworth BE, Olds TS. Development of a compendium of energy expenditures for youth. Int J Behav Nutr Phys Act. 2008;5:45. PubMed doi:10.1186/1479-5868-5-45

14. Reilly JJ, Janssen X, Cliff DP, Okely AD. Appropriateness of the definition of 'sedentary' in young children: Whole-room calorimetry study. J Sci Med Sport. 2015;18(5):565-568. PubMed doi:10.1016/j. jsams.2014.07.013

15. Sijtsma A, Corpeleijn E, Sauer PJ. Energy requirements for maintenance and growth in 3- to 4-year-olds may be overestimated by existing equations. J Pediatr Gastroenterol Nutr. 2014;58(5):642-646. PubMed doi:10.1097/MPG.0000000000000278

16. Collins CE, Burrows TL, Truby H, et al. Comparison of energy intake in toddlers assessed by food frequency questionnaire and total energy expenditure measured by the doubly labeled water method. J Acad Nutr Diet. 2013;113(3):459-463. PubMed doi:10.1016/j.jand.2012.09.021 